\title{
Kinematic Self-Similar Solutions of Locally Rotationally Symmetric Spacetimes
}

\author{
M. Sharif and M. Jamil Amir \\ Department of Mathematics, University of the Punjab, \\ Quaid-e-Azam Campus, Lahore-54590, Pakistan.
}

(Received on 28 January, 2010)

\begin{abstract}
This paper contains locally rotationally symmetric kinematic self-similar perfect fluid and dust solutions. We consider three families of metrics which admit kinematic self-similar vectors of the first, second, zeroth and infinite kinds, not only for the tilted fluid case but also for the parallel and orthogonal cases. It is found that the orthogonal case gives contradiction both in perfect fluid and dust cases for all the three metrics while the tilted case reduces to the parallel case in both perfect fluid and dust cases for the second metric. The remaining cases give self-similar solutions of different kinds. We obtain a total of seventeen independent solutions out of which two are vacuum. The third metric yields contradiction in all the cases.
\end{abstract}

Keywords: Locally rotationally symmetric, Self-similarity.

\section{INTRODUCTION}

Due to highly non-linear nature of the Einstein field equations (EFEs),

$$
R_{a b}-\frac{1}{2} g_{a b} R=8 \pi G T_{a b}
$$

the most general analytical solution has not been found till now. Although, thousands number of solutions are available in the literature but one has to impose some symmetry restrictions while solving these equations for any physical system. One of these symmetry restrictions is called isometry or Killing vector $(\mathrm{KV})$, which leads to some conservation laws [1]. There has been a recent literature [2-8, and references therein] which shows a significant interest in the study of various types of symmetry.

Self-similarity is a powerful tool to simplify the field equations. In General Relativity (GR), there does not exist any characteristic scale. A set of field equations remains invariant under a scale transformation for an appropriate matter field. This means that there exist scale invariant solutions to the EFEs, known as self-similar solutions. These solutions often play an important role in cosmological situations and gravitational collapse. The main advantage of self-similarity is that it reduces the number of independent variables by introducing a self-similar variable and hence reduces the field equations. This variable is a dimensionless combination of the independent variables, namely the space coordinates and the time. In other words, self-similarity simply reduces a system of partial differential equations to an ordinary differential equations.

In GR, many authors [9] investigated self-similar solutions for obtaining the realistic solutions of gravitational collapse. There exist several preferred geometric structures in self-similar models and one can use different approaches to study these models, such as, co-moving, homothertic, Schwarzschild approach etc. As pioneers, Cahill and Taub [10] used the co-moving approach to study self-similar solutions in the context of cosmology. In this approach, the coordinates are adopted to the fluid 4-velocity vector. In GR, self-similarity is defined by the existence of a homothetic vector (HV) field. Such similarity is called the first kind (or homothety or continuous self-similarity (CSS)). There exists a natural generalization of homothety called kinematic self- similarity defined by the existence of a kinematic self-similar (KSS) vector field.

Cahill and Taub [10] floated the idea of self-similarity in GR. They showed that it corresponds to self-similarity of the homothetic class in the context of Newtonian theory and is known as KSS of the first kind. Later, self-similarity of the second, zeroth and infinite kinds were introduced by Carter and Henriksen [11,12]. There is a great literature available [2,3,13-18] which contain several KSS perfect fluid solutions of the EFEs. It has been shown that $p=k \rho$ is the only barotropic equation of state which is compatible with self-similarity of the first kind. Carr [2] classified the most general spherically symmetric dust solutions of the EFEs admitting the self-similarity of the first kind. This work was extended by Carr and Coley [3] for all spherically symmetric perfect fluid solutions. Coley [13] concluded that the solutions in which the KSS vector is parallel to the fluid flow are necessarily Friedmann-Robertson-Walker models. McIntosh [14] proved that a vacuum spacetime admits only a nontrivial homothetic motion if the homothetic vector field is non-null and is not hypersurface orthogonal. Benoit and Coley [15] studied spherically symmetric perfect fluid solutions of the EFEs admitting KSS vector of the first, second and zeroth kinds by using analytic approach.

Sintes et al. [16] investigated the perfect fluid solutions in the case of self-similarity of the infinite kind. Carr et al. [17] considered the KSS solutions associated with the critical behavior observed in the gravitational collapse of spherically symmetric perfect fluid by using the equation of state $p=k \rho$. They also investigated solution space of self-similar spherically symmetric perfect fluid models [18] and discussed the physical aspects of these solutions. Coley and Goliath [19] studied the self-similar spherically symmetric cosmological models with a perfect fluid and a scalar field by using an exponential potential.

Maeda et al. [4,5] analyzed the KSS solution of the second kind for the titled perfect fluid case by using a relativistic polytropic equation of state. They classified the spherically symmetric perfect fluid and dust solutions admitting the KSS vector of different kinds [6] and found some interesting solutions. Sharif and Sehar [20] extended this work for cylindrically symmetric spacetimes for both perfect fluid and dust cases with tilted, parallel and orthogonal vector fields by using different equations of state. They also studied the 
physical properties of spherically [21], cylindrically [22] and plane [23] symmetric spacetimes.

Recently, Sharif and Sehar [24,25] have explored the KSS solutions of the most general plane symmetric spacetimes. Sintes [26] explored some KSS solutions of locally rotationally symmetric (LRS) spacetimes. This paper is devoted to complete the study of the KSS solutions of LRS models both for the perfect fluid and dust cases. The kinematic selfsimilar vectors of the first, second, zeroth and infinite kinds for the tilted fluid as well as parallel and orthogonal cases would be investigated.

The paper is organized as follows. In section 2, we shall give three metrics representing the non-static LRS spacetimes. Section 3 is devoted to find the KSS perfect fluid and dust solutions of different kinds for the first LRS metrics. In section 4, we shall discuss all possible KSS solutions of the second LRS metrics. The last section contains a summary and discussion of the results obtained.

\section{LOCALLY ROTATIONALLY SYMMETRIC MODELS AND KINEMATIC SELF-SIMILARITY}

Many authors [27-30] studied extensively the LRS spacetimes which contain well-known exact solutions of the EFEs. They admit a group of motions $G_{4}$ acting multiply transitively on three dimensional non-null orbits spacelike $\left(S_{3}\right)$ or timelike $\left(T_{3}\right)$ and the isotropy group is a spatial rotation. These spacetimes are represented by three families of metrics given as [26,27]

$$
\begin{aligned}
d s^{2} & =\varepsilon\left[-d t^{2}+A^{2}(t) d x^{2}\right]-B^{2}(t) d y^{2}-B^{2}(t) \Sigma^{2}(y, k) d z^{2}(2) \\
d s^{2} & =\varepsilon\left[-d t^{2}+A^{2}(t)\{d x-\Lambda(y, k) d z\}^{2}\right]-B^{2}(t) d y^{2} \\
& -B^{2}(t) \Sigma^{2}(y, k) d z^{2} \\
d s^{2} & =\varepsilon\left[-d t^{2}+A^{2}(t) d x^{2}\right]-B^{2}(t) d y^{2}-B^{2}(t) \Sigma^{2}(y, k) d z^{2}(4)
\end{aligned}
$$

where $k=-1,0,1, \varepsilon= \pm 1$,

$$
\Sigma=\left\{\begin{array}{lc}
\sin y, & k=1, \\
y, & k=0, \\
\sinh y, & k=-1,
\end{array}\right.
$$

and

$$
\Lambda= \begin{cases}\cos y, & k=1 \\ \frac{y^{2}}{2}, & k=0 \\ \cosh y, & k=-1\end{cases}
$$

The static and non-static solutions correspond to $\varepsilon=1$ and $\varepsilon=-1$ respectively. We restrict our attention towards the non-static case as the results for the static case can be obtained consequently. For $\varepsilon=-1$, the above equations take the form

$$
\begin{aligned}
d s^{2} & =d t^{2}-A^{2}(t) d x^{2}-B^{2}(t) d y^{2}-B^{2}(t) \Sigma^{2}(y, k) d z^{2},(7) \\
d s^{2} & =d t^{2}-A^{2}(t) d x^{2}-B^{2}(t) e^{2 x} d y^{2}-B^{2}(t) e^{2 x} d z^{2} \\
d s^{2} & =d t^{2}-A^{2}(t) d x^{2}-B^{2}(t) d y^{2}-\left\{A^{2}(t) \Lambda^{2}(y, k)\right. \\
& \left.+B^{2}(t) \Sigma^{2}(y, k)\right\} d z^{2}+2 A^{2}(t) \Lambda(y, k) d x d z
\end{aligned}
$$

The metrics (7) become Bianchi types $I(B I)$ or $V I I_{0}\left(B V I I_{0}\right)$ for $k=0, I I I(B I I I)$ for $k=-1$ and Kantowski-Sachs (KS) for $k=+1$. The metrics (8) represent Bianchi type $V(B V)$ or $V I I_{h}\left(B V I I_{h}\right)$ metric. The metrics (9) turn out to be Bianchi types $I I(B I I)$ for $k=0, V I I I(B V I I I)$ or $I I I(B I I I)$ for $k=-1$ and $I X(B I X)$ for $k=+1$. The energy-momentum tensor for a perfect fluid is given as

$T_{a b}=[\rho(t, y)+p(t, y)] u_{a} u_{b}+p(t, y) g_{a b}, \quad(a, b=0,1,2,3)$,

where $\rho$ is the density, $p$ is the pressure and $u_{a}$ is the fourvelocity of the fluid element in the co-moving coordinate system given as $u_{a}=(1,0,0,0)$.

A kinematic self-similar vector $\xi$ satisfies the following two conditions

$$
\begin{aligned}
£_{\xi} h_{a b} & =2 \delta h_{a b}, \\
£_{\xi} u_{a} & =\alpha u_{a},
\end{aligned}
$$

where $h_{a b}=g_{a b}-u_{a} u_{b}$ is the projection tensor, $\alpha$ and $\delta$ are constants. The similarity transformation is characterized by the scale independent ratio, $\alpha / \delta$, known as the similarity index which yields the following two cases:

$$
\text { 1. } \delta \neq 0 ; 2 . \quad \delta=0 .
$$

The case 1 yields self-similarity of the first, zeroth, second kinds and the case 2 corresponds to self-similarity of the infinite kind respectively. These are discussed below in detail.

\section{KINEMATIC SELF-SIMILAR SOLUTIONS OF THE FIRST METRIC}

For this metric, the EFEs reduce to the following form

$$
\begin{aligned}
\frac{2 \dot{A} \dot{B}}{A B}+\frac{\dot{B}^{2}}{B^{2}}+\frac{K}{B^{2}} & =\kappa \rho, \\
\frac{\ddot{A}}{A}+\frac{3 \ddot{B}}{B}+\frac{\dot{B}^{2}}{B^{2}}+\frac{\dot{A} \dot{B}}{A B}+\frac{K}{B^{2}} & =-2 \kappa p,
\end{aligned}
$$

where dot represents the differentiation w.r.t. time coordinate. The conservation of energy-momentum tensor $T^{a b}{ }_{; b}=$ 0 yields the following equation

$$
\dot{\rho}+\left(\frac{\dot{A}}{A}+\frac{2 \dot{B}}{B}\right)(p+\rho)=0, \quad p^{\prime}=0,
$$

where prime means differentiation w.r.t. $y$. In this case, the general form of a vector field $\xi$ may be given as

$$
\xi^{a} \frac{\partial}{\partial x^{a}}=h_{0}(t, y) \frac{\partial}{\partial t}+h_{2}(t, y) \frac{\partial}{\partial y},
$$

where $h_{0}$ and $h_{2}$ are arbitrary functions. When $\xi$ is parallel to the fluid flow, $h_{2}=0$ while $h_{0}=0$ indicates that $\xi$ is orthogonal to the fluid flow. When both $h_{0}$ and $h_{2}$ are non-vanishing, $\xi$ will be tilted to the fluid flow.

\subsection{Perfect Fluid Case}

Here we discuss all the above kinds when the vector field is tilted, parallel and orthogonal to the fluid flow for the perfect fluid. 


\subsubsection{Tilted Vector Field}

Case 1: For the sake of simplicity we choose $\delta$ as unity, then the KSS equations will become

$$
\begin{aligned}
\xi_{, 0}^{0} & =\alpha, \\
\xi_{, 2}^{0}-B^{2} \xi_{, 0}^{2} & =0, \\
\frac{\dot{A}}{A} \xi^{0} & =1, \\
\frac{\dot{B}}{B} \xi^{0}+\xi_{, 2}^{2} & =1, \\
\frac{\dot{B}_{B}}{B} \xi^{0}+\frac{\Sigma^{\prime}}{\Sigma} \xi_{, 2}^{2} & =1 .
\end{aligned}
$$

From Eqs.(17) to (21), we obtain the following form of $\xi^{0}$ and $\xi^{2}$

$$
\xi^{a} \frac{\partial}{\partial x^{a}}=(\alpha t+\beta) \frac{\partial}{\partial t}+c \Sigma \frac{\partial}{\partial y} .
$$

For the tilted case, the similarity index, $\alpha / \delta$, yields the following three different possibilities

(i) $\delta \neq 0, \quad \alpha=1 \quad(\beta$ can be taken to be zero $)$,

(ii) $\delta \neq 0, \quad \alpha=0 \quad$ ( $\beta$ can be taken to be unity),

(iii) $\delta \neq 0, \quad \alpha \neq 0,1 \quad$ ( $\beta$ can betaken to be zero).

These cases correspond to the self-similarity of first, zeroth and second kind respectively. We shall discuss these kinds separately.

For self-similarity of the first kind, we have $\xi^{0}=t, \xi^{2}=$ $c \Sigma$. The corresponding solution for $k=0$ takes the form

$$
\begin{aligned}
d s^{2} & =d t^{2}-a^{2} t^{2} d x^{2}-b^{2} t^{2(1-c)}\left(d y^{2}+y^{2} d z^{2}\right), \\
\rho & =\frac{9}{16 \kappa t^{2}}=-3 p
\end{aligned}
$$

where $a$ and $b$ are arbitrary constants but $c=\frac{1}{2}, 1,2$. There exists no solution for $k= \pm 1$.

Self-similarity of the zeroth kind yields $\xi^{0}=1, \xi^{2}=c \Sigma$ and the solution for $k=0$ turns out to be

$$
\begin{aligned}
d s^{2} & =d t^{2}-a^{2} e^{2 t} d x^{2}-b^{2} e^{2(1-c) t}\left(d y^{2}+y^{2} d z^{2}\right), \\
\rho & =\frac{1}{\kappa}(c-1)(c-3), \quad p=\frac{-1}{2 \kappa}\left(4 c^{2}-9 c+6\right) .
\end{aligned}
$$

Here $c=0, \frac{1}{2}, \frac{3}{2}$ and no solution exists for $k= \pm 1$.

In the second kind, $\xi^{0}=\alpha t, \xi^{2}=c \Sigma$ and the corresponding solution for $k=0$ becomes

$$
\begin{aligned}
d s^{2} & =d t^{2}-a^{2} t^{2 / \alpha} d x^{2}-b^{2} t^{2(1-c) / \alpha}\left(d y^{2}+y^{2} d z^{2}\right), \\
\rho & =\frac{1}{\kappa \alpha^{2} t^{2}}(c-1)(c-3), \\
p & =\frac{-1}{2 \kappa \alpha^{2} t^{2}}\left(4 c^{2}-9 c+6+3 \alpha c-4 \alpha\right),
\end{aligned}
$$

where $c$ is either zero or $\frac{1}{2}, \frac{3-\alpha}{2}$. There does not exist any solution for $k= \pm 1$.
Case 2: For $\delta=0$ and $\alpha \neq 0$ ( $\alpha$ can be unity and $\beta$ can be rescaled to zero), the self-similarity is known as infinite kind. In this case, the KSS equations take the following form

$$
\begin{aligned}
\xi_{, 0}^{0} & =1, \\
\xi_{, 2}^{0}-B^{2} \xi_{, 0}^{2} & =0, \\
\dot{A}_{\xi^{0}} & =0, \\
\frac{\dot{B}}{B} \xi^{0}+\xi_{, 2}^{2} & =0, \\
\frac{\dot{B}_{\xi^{0}}+\frac{\Sigma^{\prime}}{\Sigma} \xi_{, 2}^{2}}{\xi^{2}} & =0 .
\end{aligned}
$$

Here $\xi^{0}=t+c_{0}, \xi^{2}=c \Sigma$ and the corresponding solution for $k=0$ is

$$
\begin{aligned}
d s^{2} & =d t^{2}-a^{2} d x^{2}-b^{2}\left(t+c_{0}\right)^{-2 c}\left(d y^{2}+y^{2} d z^{2}\right), \\
\rho & =\frac{c^{2}}{\kappa\left(t+c_{0}\right)^{2}}, \quad p=\frac{-c(4 c+3)}{2 \kappa\left(t+c_{0}\right)^{2}},
\end{aligned}
$$

where $c=0,-\frac{1}{2}$. It is mentioned here that the solutions become vacuum and stiff fluid for $c=0$ and $c=-\frac{1}{2}$ respectively. The solution for $k=+1$ is given by

$$
\begin{aligned}
d s^{2} & =d t^{2}-a^{2} d x^{2}-b^{2}\left(d y^{2}+\sin ^{2} y d z^{2}\right), \\
\rho & =-p / 2 .
\end{aligned}
$$

The solution for $k=-1$ is

$$
\begin{aligned}
d s^{2} & =d t^{2}-a^{2} d x^{2}-b^{2}\left(d y^{2}+\sinh ^{2} y d z^{2}\right), \\
\rho & =-p / 2 .
\end{aligned}
$$

It is noted that $\xi^{2}$ vanishes for $k= \pm 1$, i.e., these solutions fall in the parallel case.

\subsubsection{Parallel Vector Field}

Case 1: In this case $\xi^{1}, \xi^{2}, \xi^{3}=0$ and the KSS equations for $\delta=1$ take the form

$$
\begin{aligned}
& \xi_{, 0}^{0}=\alpha, \\
& \frac{\dot{A}}{A} \xi^{0}=1, \\
& \frac{\dot{B}}{B} \xi^{0}=1 .
\end{aligned}
$$

Integrating Eq.(34), we obtain

$$
\xi^{0}=(\alpha t+\beta) .
$$

For self-similarity of the first kind, $\xi^{0}=t$ and the corresponding solution for $k=0$ becomes

$$
\begin{aligned}
d s^{2} & =d t^{2}-a^{2} t^{2} d x^{2}-b^{2} t^{2}\left(d y^{2}+y^{2} d z^{2}\right), \\
\rho & =\frac{3}{\kappa t^{2}}=-3 p .
\end{aligned}
$$

In the zeroth kind, $\xi^{0}=1$ and the corresponding solution for $k=0$ is

$$
\begin{aligned}
d s^{2} & =d t^{2}-a^{2} e^{2 t} d x^{2}-b^{2} e^{2 t}\left(d y^{2}+y^{2} d z^{2}\right), \\
\rho & =\frac{3}{\kappa}=-p .
\end{aligned}
$$


Self-similarity of the second kind, $\xi^{0}=\alpha t$, yields the following solution for $k=0$

$$
\begin{aligned}
d s^{2} & =d t^{2}-a^{2} t^{2 / \alpha} d x^{2}-b^{2} t^{2 / \alpha}\left(d y^{2}+y^{2} d z^{2}\right), \\
\rho & =\frac{3}{\kappa \alpha^{2} t^{2}}, \quad p=-\frac{1}{\kappa \alpha^{2} t^{2}}(3-2 \alpha) .
\end{aligned}
$$

There exists no solution for $k= \pm 1$ in the above kinds.

Case 2: The infinite kind, $\delta=0, \alpha \neq 0$ yields the same solution as given in Eq.(31) for $c=0$. The solution for $k= \pm 1$ are the same as given by Eqs.(32) and (33).

\subsubsection{Orthogonal Vector Field}

There does not exist any solution for the perfect fluid case when the vector field is orthogonal to the fluid flow.

\subsection{Dust Case}

For the dust case, we take $p=0$ in Eqs.(14) and (15) so that

$$
\begin{gathered}
\frac{\ddot{A}}{A}+\frac{3 \ddot{B}}{B}+\frac{\dot{B}^{2}}{B^{2}}+\frac{\dot{A} \dot{B}}{A B}+\frac{K}{B^{2}}=0, \\
\dot{\rho}+\left(\frac{\dot{A}}{A}+\frac{2 \dot{B}}{B}\right) \rho=0,
\end{gathered}
$$

The KSS solutions for the tilted case are given in table 1

Table 1. KSS solutions for the tilted dust case when $k=0$

\begin{tabular}{|l|l|l|l|l|}
\hline Kinds & $\mathbf{A}(\mathbf{t})$ & $\mathbf{B}(\mathbf{t})$ & Density & KSS Vectors \\
\hline First Kind & $a t$ & $b, b t^{1 / 2}$ & 0 & $\xi^{0}=t, \quad \xi^{2}=y$ \\
\hline Zeroth Kind & - & - & - & - \\
\hline Second Kind & $a t^{-\frac{1}{3}}$ & $b t^{\frac{2}{3}}$ & 0 & $\xi^{0}=-3 t, \quad \xi^{2}=3 y$ \\
\hline Infinite kind & $a$ & $b$ & 0 & $\xi^{0}=t+c_{0}, \quad \xi^{2}=0$ \\
\hline
\end{tabular}

Here $a, b$ and $c_{0}$ are arbitrary constants. The solution for the infinite kind will fall in the parallel dust case as $\xi^{2}$ vanishes for this case. The KSS solutions for the parallel dust case, when $\xi^{2}=0$, are given in table 2

Table 2. KSS solutions for the parallel dust case when $k=0$

\begin{tabular}{|l|l|l|l|l|}
\hline Kinds & $\mathbf{A}(\mathbf{t})$ & $\mathbf{B}(\mathbf{t})$ & Density & KSS Vectors \\
\hline First Kind & - & - & - & - \\
\hline Zeroth Kind & - & - & - & - \\
\hline Second Kind & $a t^{\frac{2}{3}}$ & $b t^{\frac{2}{3}}$ & $\frac{4}{3 \kappa t^{2}}$ & $\xi^{0}=\frac{3}{2} t$ \\
\hline Infinite kind & $a$ & $b$ & 0 & $\xi^{0}=t+c_{0}$ \\
\hline
\end{tabular}

There exists no solution in all the cases mentioned in the tables 1 and 2 for $k= \pm 1$. Further, there exists no solution for the dust case when the vector field is orthogonal to the fluid flow.

\section{KINEMATIC SELF-SIMILAR SOLUTIONS OF THE 2ND METRIC}

For this metric (8), the EFEs reduce to the following form

$$
\begin{gathered}
\frac{2 \dot{A} \dot{B}}{A B}+\frac{\dot{B}^{2}}{B^{2}}-\frac{3}{A^{2}}=\kappa \rho, \\
\frac{\ddot{A}}{A}+\frac{3 \ddot{B}}{B}+\frac{\dot{B}^{2}}{B^{2}}+\frac{\dot{A} \dot{B}}{A B}-\frac{2}{A^{2}}=-2 \kappa p, \\
\frac{\dot{A}}{A}-\frac{\dot{B}}{B}=0 .
\end{gathered}
$$

The conservation of energy-momentum tensor $T^{a b}{ }_{; b}=0$ yields the same equation as given by Eq.(16). Here prime means differentiation w.r.t. $x$. For these spacetimes, the general form of a vector field $\xi$ may be given as

$$
\xi^{a} \frac{\partial}{\partial x^{a}}=h_{0}(t, x) \frac{\partial}{\partial t}+h_{1}(t, x) \frac{\partial}{\partial x},
$$

where $h_{0}$ and $h_{1}$ are arbitrary functions.

\subsection{Perfect Fluid Case}

Now we shall discuss the cases (1) and (2) when the vector field is tilted, parallel and orthogonal to the fluid flow.

\subsubsection{Tilted Vector Field}

This case reduces to the parallel perfect fluid case.

\subsubsection{Parallel Vector Field}

Case 1: For the parallel vector field, we take $\xi^{1}=0$ and $\delta=1$ and the KSS equations imply that $\xi^{0}=\alpha t+\beta$. For the self-similarity of the first kind, $\xi^{0}=t$ and the corresponding solution take the form

$$
\begin{aligned}
d s^{2} & =d t^{2}-a^{2} t^{2} d x^{2}-b^{2} t^{2} e^{2 x}\left(d y^{2}+d z^{2}\right), \\
\rho & =-\frac{3}{\kappa t^{2}}\left(\frac{1}{a^{2}}-1\right)=-3 p .
\end{aligned}
$$

In self-similarity of the zeroth kind, $\xi^{0}=1$ and the corresponding solution is

$$
\begin{aligned}
d s^{2} & =d t^{2}-a^{2} e^{2 t} d x^{2}-b^{2} e^{2(t+x)}\left(d y^{2}+d z^{2}\right), \\
\rho & =\frac{3}{\kappa}\left(1-\frac{1}{a^{2}} e^{-2 t}\right)=-p .
\end{aligned}
$$

For the second kind, $\xi^{0}=\alpha t$, the solution becomes

$$
\begin{aligned}
d s^{2} & =d t^{2}-a^{2} t^{2 / \alpha} d x^{2}-b^{2} t^{2 / \alpha} e^{2 x}\left(d y^{2}+d z^{2}\right), \\
\rho & =\frac{3}{\kappa}\left(\frac{1}{a^{2} t^{2}}-\frac{1}{a^{2} t^{\frac{2}{\alpha}}}\right), \quad p=-\rho+\frac{2}{\kappa \alpha t^{2}} .
\end{aligned}
$$

Case 2: The infinite kind, $\delta=0, \alpha \neq 0$, leads to $\xi^{0}=t+$ $c_{0}, A=a, B=b$ and the corresponding solution turns out to be

$$
\begin{aligned}
d s^{2} & =d t^{2}-a^{2} d x^{2}-b^{2} e^{2 x}\left(d y^{2}+d z^{2}\right), \\
\rho & =-\frac{3}{\kappa a^{2}}=-3 p .
\end{aligned}
$$




\subsubsection{Orthogonal Vector Field}

Here all the possibilities lead to contradiction.

\subsection{Dust Case}

For the dust case, we take $p=0$ in Eqs.(14) and (15) so that

$$
\begin{gathered}
\frac{\ddot{A}}{A}+\frac{3 \ddot{B}}{B}+\frac{\dot{B}^{2}}{B^{2}}+\frac{\dot{A} \dot{B}}{A B}+\frac{K}{B^{2}}=0, \\
\dot{\rho}+\left(\frac{\dot{A}}{A}+\frac{2 \dot{B}}{B}\right) \rho=0,
\end{gathered}
$$

The tilted dust case reduces to the parallel dust case. The KSS solutions for the parallel case, when $\xi^{1}=0$, are given in table 3 .

Table 3. KSS solutions for the parallel dust case

\begin{tabular}{|l|l|l|l|l|}
\hline Kinds & A(t) & B(t) & Density & KSS Vectors \\
\hline First Kind & \pm 1 & $b$ & 0 & $\xi^{0}=t$ \\
\hline Zeroth Kind & - & - & - & - \\
\hline Second Kind & - & - & - & - \\
\hline Infinite kind & - & - & - & - \\
\hline
\end{tabular}

The dust orthogonal case yields contradiction for all the possibilities. Also, we obtain contradiction in all the cases of the third metric (9).

\section{CONCLUSION}

We have investigated the LRS spacetimes which admit self-similarity of the first, zeroth, second and infinite kinds for both perfect fluid and dust cases. We have explored the possibilities when KSS vector is tilted, parallel or orthogonal to the fluid flow. We find a total of seventeen independent KSS solutions out of which two are vacuum.

For the metric (7), there arise three KSS solutions in the tilted perfect fluid case and coincide with the results given by Sintes [25] for $n=0$ and $m=c$ in the first, zeroth and second kinds. For the infinite kind, we find three solutions which do not agree with the solutions given in [25]. It is mentioned here that the KSS solutions of the first kind turns out to be the radiation case and the tilted perfect fluid case of the infinite kind reduces to the parallel perfect fluid case for $k= \pm 1$. The parallel perfect fluid case gives three independent KSS solutions in the first, zeroth and second kinds. These solutions also coincide with those given in [25]. The infinite kind yields the same solutions as for the tilted perfect fluid case of the infinite kind when $c=0$. For the tilted dust case, we have two KSS solutions of the first kind and one of the second kind which are vacuum. The infinite kind gives the same solution as given in Eq.(31) for $c=0$ while the zeroth kind gives no solution. In the parallel dust case, the first and zeroth kinds give contradiction while the infinite kind gives the same solution as given in Eq.(31) for $c=0$. We obtain one independent solution in the second kind. The orthogonal case yields contradiction both in prefect fluid and dust cases.

For the metric (8), the tilted perfect fluid case reduces to the parallel case and this yields four KSS solutions of the first, zeroth, second and infinite kinds which coincide with [25] when $n=0$ and $m=c$. In the dust case, there exists only one KSS solution of the first kind which coincides with Eq.(50) for $a= \pm 1$ while the second, zeroth and infinite kinds yield contradiction. It is mentioned here that the orthogonal case always gives contradiction.

For the metric (9), we have contradiction in all the cases. The summary of the results is given in the following tables.

Table 4. Perfect fluid kinematic self-similar solutions of the metric (7)

\begin{tabular}{|l|l|}
\hline Self-similarity & Solution \\
\hline First kind (tilted) & Solution given by Eq.(23) \\
\hline First kind (parallel) & Solution given by Eq.(38) \\
\hline First kind (orthogonal) & None \\
\hline Zeroth kind (titled) & Solution given by Eq.(24) \\
\hline Zeroth kind (parallel) & Solution given by Eq.(39) \\
\hline Zeroth kind (orthogonal) & None \\
\hline Second kind (tilted) & Solution given by Eq.(25) \\
\hline Second kind (parallel) & Solution given by Eq.(40) \\
\hline Second kind (orthogonal) & None \\
\hline Infinite kind (tilted) & Solution given by Eq.(31) \\
\hline Infinite kind (parallel) & $\begin{array}{l}\text { Solution given by Eqs.(33),(31) and } \\
\text { (32) for } k=-1,0,1 \text { respectively }\end{array}$ \\
\hline Infinite kind (orthogonal) & None \\
\hline
\end{tabular}

Table 5. Dust kinematic self-similar solutions of the metric (7) 


\begin{tabular}{|l|l|}
\hline Self-similarity & Solution \\
\hline First kind (tilted) & Two vacuum solutions given in table 1 \\
\hline First kind (parallel) & None \\
\hline First kind (orthogonal) & None \\
\hline Zeroth kind (titled) & None \\
\hline Zeroth kind (parallel) & None \\
\hline Zeroth kind (orthogonal) & None \\
\hline Second kind (tilted) & Vacuum solution given in table 1 \\
\hline Second kind (parallel) & Solution given in table 2 \\
\hline Second kind (orthogonal) & None \\
\hline Infinite kind (tilted) & $\begin{array}{l}\text { Vacuum solution given by Eq.(31) } \\
\text { for } c=0\end{array}$ \\
\hline Infinite kind (parallel) & $\begin{array}{l}\text { Vacuum solution given by Eq.(31) } \\
\text { for } c=0\end{array}$ \\
\hline Infinite kind (orthogonal) & None \\
\hline
\end{tabular}

Table 6. Perfect fluid kinematic self-similar solutions of the metric (8)

\begin{tabular}{|l|l|}
\hline Self-similarity & Solution \\
\hline First kind (tilted) & None \\
\hline First kind (parallel) & Solution given by Eq.(47) \\
\hline First kind (orthogonal) & None \\
\hline Zeroth kind (titled) & None \\
\hline Zeroth kind (parallel) & Solution given by Eq.(48) \\
\hline Zeroth kind (orthogonal) & None \\
\hline Second kind (tilted) & None \\
\hline Second kind (parallel) & Solution given by Eq.(49) \\
\hline Second kind (orthogonal) & None \\
\hline Infinite kind (tilted) & None \\
\hline Infinite kind (parallel) & Solution given by Eq.(50) \\
\hline
\end{tabular}

We would like to mention here that Eqs.(38), (39) and (40) are the special cases of Eqs.(23), (24) and (25) respectively for $c=0$. Further, Eqs.(23) and (24) represent orthogonal spatially homogeneous perfect fluid Bianchi $I$ models with homothetic vector field and the equation of state with $\gamma=\frac{2}{3}$ and $\gamma=0$ respectively. These can correspond to FRW models under particular coordinate transformations. Similarly, Eqs.(47) and (48) represent the orthogonal spatially homogeneous perfect fluid Bianchi $V$ models with homothetic vector field and the equation of state with $\gamma=\frac{2}{3}$ and $\gamma=0$ respectively. Also, Eq.(31) represents Minkowski space for $c=0$. The dust parallel case of the first kind yields vacuum solution as a special case given by Eq.(50).

\section{Acknowledgment}

We appreciate and acknowledge the Higher Education Commission Islamabad, Pakistan for its financial support through the Indigenous PhD 5000 Fellowship Program Batch-I.
[1] Noether, E. Nachr, Akad. Wiss. Gottingen, II, Math. Phys. K12(1918)235;

Davis, W.R. and Katzin, G.H. Am. J. Phys. 30(1962)750;

Petrov, A.Z.: Einstein Spaces (Pergamon, Oxford University Press, 1969);

Hojman, L. Nunez, 1. Patino, A. and Rago, H.: J. Math. Phys. 27(1986)281.

[2] Carr, B.J.: Phys. Rev. D62(2000)044022.

[3] Carr, B. J. and Coley, A. A.: Phys. Rev. D62(2000)044023.

[4] Maeda, H., Harada, T., Iguchi, H. and Okuyama, N.: Phys. Rev. D66(2002)027501.

[5] Maeda, H., Harada, T., Iguchi, H. and Okuyama, N.: Prog. Theor. Phys. 108(2002)819.

[6] Maeda, H., Harada, T., Iguchi, H. and Okuyama, N.: Prog. Theor. Phys. 110(2003)25.

[7] Sharif, M.: J. Math. Phys. 44(2003)5141; ibid 45(2004)1518; ibid 45(2004) 1532.

[8] Sharif, M. and Aziz, S.: Int. J. Mod. Phys. D14(2005)1527.

[9] Penston, M.V.: Mon. Not. R. Astr. Soc. 144(1969)425; Larson, R.B.: Mon. Not. R. Astr. Soc. 145(1969)271; Shu, F.H.: Astrophys. J. 214(1977)488; Hunter, C.: Astrophys. J. 218(1977)834.
[10] Cahill, M. E. and Taub, A. H.: Commun. Math. Phys. 21(1971)1.

[11] Carter, B. and Henriksen, R. N.: Annales De Physique 14(1989)47.

[12] Carter, B. and Henriksen, R. N.: J. Math. Phys. 32(1991)2580.

[13] Coley, A. A.: Class. Quantum Grav. 14(1997)87.

[14] McIntosh, C.B.G.: Gen. Rel. Gravit. 7(1975)199.

[15] Benoit, P. M. and Coley, A. A.: Class. Quantum Grav. 15(1998)2397.

[16] Sintes, M.A., Benoit, P.M. and Coley, A. A.: Gen. Relativ. Gravit. 33(2001)1863.

[17] Carr, B. J., Coley, A. A., Golaith, M., Nilsson, U. S. and Uggla, C.: Class. Quantum Grav. 18(2001)303.

[18] Carr, B. J., Coley, A. A., Golaith, M., Nilsson, U. S. and Uggla, C.: Phys. Rev. D61(2000)081502.

[19] Coley, A. A. and Golaith, M.: Class. Quantum Grav. 17(2000) 2557.

[20] Sharif, M. and Aziz, S.: Int. J. Mod. Phys. D14 (2005)1527.

[21] Sharif, M. and Aziz, S.: Int. J. Mod. Phys. D14 (2005)73.

[22] Sharif, M. and Aziz, S.: Int. J. Mod. Phys. A20(2005)7579.

[23] Sharif, M. and Aziz, S.: J. Korean Phys. Soc. 47(2005)757.

[24] Sharif, M. and Aziz, S.: J. Korean Phys. Soc. 49(2006)21. 
[25] Sharif, M. and Aziz, S.: Class. Quantum Grav. 24(2007)605.

[26] Sintes, M.A.: Class. Quantum Grav. 15(1998)3689.

[27] Ellis, G.F.R.: J. Math. Phys. 8(1967)1171.

[28] Steward, J.M. and Ellis, G.F.R.: J. Math. Phys. 9(1968)1072.

[29] Ellis, G.F.R. and MacCallum, M.A.H.: Commun. Math. Phys.
12(1969)108.

[30] Tsamparlis, M. and Apostolopoulos, P.S.: Gen. Relativ. Gravit. 36(2004)47. 